\title{
Chronic osteomyelitis in Sub-Saharan Africa-A Review
}

\author{
Kubwimana Olivier ${ }^{*}$, Uwizeyimana Eugene ${ }^{1}$, Legg Lynn ${ }^{2}$ and Lucero-Prisno Don Eliseo III $^{3}$ \\ ${ }^{1}$ Department of Orthopaedic Surgery, School of Medicine and Pharmacy, University of Rwanda, Kigali, Rwanda \\ ${ }^{2}$ Institute of Cardiovascular \& Medical Sciences, School of Medicine, Dentistry and Nursing, University of Glasgow, Glasgow \\ ${ }^{3}$ Department of Global Health and Development, London School of Hygiene and Tropical Medicine, London, UK
}

\begin{abstract}
Background: Chronic osteomyelitis (COM) has remained an orthopaedic clinical challenge for many decades. It poses a socio-economic burden to patients and the health care system. We conducted this study to understand the epidemiological and clinical features of COM in Sub-Saharan Africa (SSA).

Methods: A database-based search was done through PubMed, Google Scholar and Bioline for articles focusing on the epidemiology and clinical features of COM in SSA. Thirty-three articles were considered in the study based on the standards of assessing the level of evidence. Analysis and synthesis of the articles were conducted.

Results: The incidence of COM in SSA is far greater than high income countries. Tibia is the commonly affected bone with more than $30 \%$ of cases, while the peak age is between the interval 10-21 years of age. Sickle cell disease, peripheral vascular disease, diabetes mellitus, Buluri ulcers and septicemia were reported as potential risk factors in SSA. 50- 93\% of patients present with sinus drainage whereas Staphylococcus Aureus is the causative agent in $60-80 \%$ of the cases. Standard radiography and $\mathrm{CT}$ scan remain useful diagnostic tools in this region. The curative therapy involves radical surgical infection control with adjunct reconstructive procedures and antibiotherapy.

Conclusion: COM remains on the rise in SSA. This trend is in line with the rise in exogenous osteomyelitis, road traffic injuries in addition to factors like patient characteristics, ignorance and poverty. Adolescents are more affected where immunosuppressive and vaso-occlusive disorders are main risk factors. We recommend extension of research on COM to overcome scarcity of data seen.
\end{abstract}

\section{Background}

Osteomyelitis is a gradual, progressive, inflammatory process involving the bone cortex and its marrow. Negligence or suboptimal management evolve and progress to chronic and long-term disease. Eventually the primary bone will undergo necrosis and form a sequestrum whereas the newly formed, reactive bone is called an involucrum [1,2]. Chronic osteomyelitis is defined as bone infection lasting 6 weeks or more with radiological evidences [3]. The last several decades witnessed a substantial rise in osteomyelitis rate despite advanced diagnostic and treatment modalities; this rise was associated with major shifting in causes and risk factors of osteomyelitis where posttraumatic osteomyelitis was predominated highly marked [4]. Almost 7 decades ago osteomyelitis was considered as a lethal condition and the patients who survived had severe comorbidities. The significant decline in osteomyelitis mortality and morbidity was attributed to optimal use of antibiotherapy and advanced surgical techniques [5]. COM not only causes long term disability, it can on occasion cause other malignant conditions, notably squamous cell carcinoma (Marjolin's ulcer), arising from the long-standing sinus drainage and chronic inflammation with a progression to metaplasia, dysplasia, and neoplasia over time [6,7].

The USA witnessed an exponential rise of osteomyelitis in the elderly population from 1969 to 2009 attributed to the dramatic rise in diabetes mellitus [8]. COM can present in various types from well localized to diffuse. The early course of the disease is marked by its acute and subacute phases. Timely and optimal management in this early phase of disease prevent progression to COM with the attendant morbidity and unproductivity for long periods. Misdiagnosis was identified as the main medical challenge at primary health facilities keeping COM on its rise in resource constrained settings, whereas ignorance and financial problems rank on the top of socio-economic factors [9]. The African and global research databases present a scarcity of data related to general consensus of chronic osteomyelitis in SubSaharan Africa. We therefore undertook this study to update the epidemiology, main clinical features, and diagnostic and treatment modalities of chronic osteomyelitis in SSA.

\section{Objectives}

\section{Main objectives}

- Assess the epidemiology and clinical presentation of chronic osteomyelitis in SSA.

- Highlight currently available diagnostic and therapeutic modalities for COM in SSA.

\section{Specific objectives}

- Report general incidence, commonly affected bones, and mean age of COM in SSA

*Correspondence to: Kubwimana Olivier, Department of Orthopaedic Surgery, School of Medicine and Pharmacy, University of Rwanda, Kigali, Rwanda, E-mail: oliviee3@gmail.com

Key words: Osteomyelitis, long bones, orthopedics, epidemiology, Sub-Saharan Africa

Received: April 11, 2019; Accepted: April 25, 2019; Published: April 29, 2019 
- Document risk factors of osteomyelitis reported in SSA.

- Note signs and symptoms of COM in SSA.

- Report diagnostic and therapeutic modalities available in SSA.

\section{Search methodology}

A computer-based search for PubMed, Google Scholar and Bioline International databases was performed unrestrictedly targeting articles published between January 1998 and November 2018 focusing on epidemiology, risk factors, clinical presentation, diagnostic and treatment modalities. Our search was guided with the following key terms in various combinations "epidemiology AND osteomyelitis"; "prevalence of osteomyelitis AND Africa"; "causes AND osteomyelitis"; "Epidemiology of chronic osteomyelitis in Africa"; "risk factors of long bone osteomyelitis in Africa "; causes of chronic osteomyelitis in Africa"; "Prevalence of osteomyelitis"; "risk factors AND osteomyelitis"; "osteomyelitis OR clinical features"; "osteomyelitis in Africa AND treatment"; "diagnosis AND osteomyelitis", and "management AND diagnosis OR bone infection". There was no geographical restriction of studies (when reporting SSA findings). We aimed to retain studies with a relatively higher level of evidence. A standard method of grading the levels of evidence was applied; each article was classified utilizing nomenclature from the Agency for Healthcare Research and Quality, (appendix 1) [10].

Articles retrieved by each search were reviewed by titles and abstract and when pertinent, the article was opened for full text review and downloaded to a separate folder. Each of the three databases were searched for articles published in English as priority, but we also included a minority of studies with pertinent findings published in French. These studies were translated in English. The first articles brought were best matching; studies not matching the topic were immediately rejected. Selected articles were analyzed by two independent reviewers who extracted data which were summarized. Co-reviewers then reviewed the selected articles to create a consensus for inclusion in the final review. Reviewers focused on ascertaining the incidence of osteomyelitis in Africa. The range of incidences were recorded in SSA and compared to the rest of the world to identify any differences. Risk factors and comorbidities associated with chronic osteomyelitis were recorded. We assessed, highlighted and documented what we consider the preferred current diagnostic and treatment modalities available in SSA.

\section{Results}

\section{Incidence of COM in Sub-Saharan Africa}

Acute and subacute osteomyelitis are initial disease stages; delay in diagnosis or suboptimal management result in devastating morbidity including chronic osteomyelitis which is quite common and mainly restricted to low- and middle-income countries, although it does occur in high-income countries as well. The documented incidence of paediatric osteomyelitis in HICs is $1.94-13 / 100000$ in contrast to reported incidence of 43-200/100000 reported in LICs globally, likely reflecting the higher incidence in Sub-Saharan Africa [11]. The SSA population is young in comparison to the HICs. A large proportion of the paediatric population reside in rural areas and face possible poverty and malnutrition, a milieu where eventually COM can arise [12]. Anatomically, osteomyelitis can occur nigh anywhere, however, given the functions, vulnerability of different part of the body, and soft tissue coverage of the bone, the tibia (31-35\%) remains the leading bone affected by COM while the femur ranks second $[13,14]$. COM has reported to have its peak incidence in the second decade of life between
10- 21 years of age, with a male to female ratio ranging to $1.2-2.7 / 1$ [5,13-16]. In our region despite increases of osteo-synthetic material use for fracture treatment, $80 \%$ of osteomyelitis is hematogenous [5]. Patients with chronic osteomyelitis mainly consult for continuous pus drainage or limb deformity; hence, they usually present to the outpatient Department. A hospital based multicentre study in Uganda reported COM to account near $10 \%$ of orthopaedic outpatient consults and $8 \%$ of paediatric admission $[13,17]$.

\section{Risk factors associated with COM}

Bahebeck J et al. [18] in their study with a relatively higher level of evidence, documented sickle cell disease (SCD) (Hemoglobin SS or SC) as a risk factor for chronic osteomyelitis. In patients with SCD and a suspicion of locomotor disorders, $17 \%$ were identified with chronic osteomyelitis [18]. Osteo-synthetic implants in situ become covered by a coat of protein increasing the risk of becoming infected; thus, open reduction and internal fixation utilizing an implant is a risk factor for developing chronic osteomyelitis [19]. Peripheral vascular diseases (PVD) impair wound healing and increase the risk of chronic osteomyelitis. In addition to PVD, Hatzenbuehler et al. [20] identified diabetes mellitus to increase the risk of chronic osteomyelitis. Ilorin found a significant increase of hematogenous chronic osteomyelitis in patients with clinical and laboratory features of confirmed septicaemia [5]. Mycobacterium ulcerans causes Buruli ulcers mainly in resourceconstrained settings, notably in Sub-Saharan Africa. Buluri ulcers have been linked with $\mathrm{COM}$ at $60 \%$ where bones beneath the ulcers are likely to suffer most [21]. In SSA along with worldwide patients with sickle cell disease suffer mostly from salmonella COM [22,23]. SSA reports document a polymicrobial COM in more than $40 \%$ of cases. Enterobacteriaceae and Staphylococcus aureus remained the most usually isolated bacteria, moreover gram-negative cases were more prevalent than previously reported. The above trademark of unusual causative agent was substantially resistant to most of broadspectrum antibiotics locally used, notably Enterobacteriaceae was resistant at 70\% [24]. The large Sub-Saharan area is dominantly rural, hence a substantial number of the population live in remote areas and do not access effectively the health care system, hence a likelihood immunosuppression notably due to malnutrition, moreover initial tradition healers consultation delay patient to reach hospitals in early stage of the disease [25-27]. In terms of causative organisms normal skin flora bacteria were found to be most commonly involved, Staphylococcus aureus is the leading microorganism $[1,12,28]$ at 60 $80 \%[12,14,29]$, followed respectively in decreasing order by Klebsiella and Pseudomonas species.(29)

\section{Clinical features of COM in Sub-Saharan Africa}

The hallmark of the clinical presentation lies in its chronicity with signs and symptoms persisting despite the use of antibiotics. A sinus discharging pus is the predominant clinical feature, bone pain, limb deformity, and pathological fractures present in many COM patients [29]. Paediatric patients have a relatively higher risk of long-term morbidity. They often present with similar clinical features as adults; they are at risk of developing bone overgrowth and joint stiffness [12] One SSA center reported a sinus in 50-93\% [7,29]. Patients presented with pain and swelling of the limb (54\%) and functional impairment of a limb was noted in $30 \%$ of patients presenting with chronic osteomyelitis [7].

\section{Diagnosis of COM in Sub-Saharan Africa}

As with other conditions, one reaches the correct diagnosis in $\mathrm{COM}$ by pursuing a systematic evaluation and workup. It commences 
with a thorough history taking, retrieving the onset and progressive course of the disease, prior operative history, notably any instances of fracture care with or without the use of prosthetic material. A careful, thorough physical exam follows with a focus on the affected extremity [30].

A combination of laboratory investigations and standard radiographic examinations point to the real diagnosis; the later may reveal evidence of Brodie's abscesses, sequestrum and formation of involucrum, foreign bodies, orthopaedic implants, etc [31]. Different imaging modalities play a considerable role in diagnosing COM. Although ultrasound waves do not cross the bone cortex, it remains a useful tool when looking for periosteal reaction or guiding needle aspiration. Standard X-ray and CT imaging remain the gold standards to localize dead bone. MRI, if available and affordable, is advised (versus 'useful') prior to radical sequestrectomy and debridement as it well identifies extension to soft tissues [30]. Standard radiography remains a useful investigative tool. Imaging findings of osteomyelitis are classified into 3 types: A as Brodie's type, B with 4 subtypes as sequestrum and involucrum: (B1: localized cortical sequestrum; B2: sequestrum with structural involucrum; B3: sequestrum with sclerotic involucrum; B4: sequestrum without structural involucrum), and type $C$ a sclerotic type [12]. Prior to operation, thorough physiological status assessment has to be carried out by assessing the haemato-biochemical and nutritional workup and address potential modifiable risk factors of SSI in order to improve and promote a better outcome [30].

\section{Treatment of COM}

Chronic osteomyelitis doesn't always appear as black and white; it may be misdiagnosed as a musculoskeletal neoplastic condition and eventually negatively impact the outcome, hence upon suspicion of acute or subacute osteomyelitis optimal management may significantly reduce progression to COM [32]. Pitfalls in management relate to surgical technique and a high remission rate at 1year [28]. A very good outcome at $90 \%$ of COM cases is warranted when a multidisciplinary management is opted, where there is a joint effort between, surgical, paramedical personnel and nutritionists [26]. COM management in SSA remains challenging, curative management is often the first option, however in particular cases when it comes to an expected poor surgical outcome palliation treatment can be done by chronic suppressive antibiotherapy alone. The mainstays of curative management in SSA as elsewhere include infection control, effective dead space management, bony and soft tissues reconstructive procedures, and longstanding appropriate antibiotics $[3,30]$.

\section{Surgical infection control}

Surgical infection control is achieved by aggressive debridement, abscess drainage, curettage, bone drilling and sequestrectomy. The procedure should be properly and timely planned to limit serial reconstructive procedures $[3,7,12,26]$. COM is a long-term surgical morbidity which is very difficult to manage where sequestrum as dead bone contains pockets of purulence therefore antibiotics serve as adjunctive, not curative agents since they do not penetrate dead tissues. Thus, sequestrectomy is the standard treatment but needs to be thorough. The loco-regional data report good outcomes when strong involucrum withstanding body weight is formed in case of appendicular skeleton involvement; lacking a robust involucrum predisposes to a postoperative risk of facture which may be prevented with application of a splint or skeletal tractions [13,31]. In Uganda, sequestrectomy was the surgical intervention performed for COM $60 \%$ of the time [13].

\section{Dead space management}

In resource constrained areas dead space management utilizing inoculation of locally made calcium sulfate impregnated antibiotics (such as ceftriaxone) with PMMA (define) beads in bone cavity after thorough sequestrectomy and debridement has proven to be effective in chronic osteomyelitis in ridding remnant infection [27,33]. A closed suctioning drainage system post sequestrectomy known as the Lautenbach procedure is also effective in the obliteration of dead space in COM [3].

\section{Reconstructive phase}

A stepwise approach in the management of COM is of an utmost importance. Radical infection control is initiated and then followed by serial bony and soft tissues reconstructive procedures. On occasion amputation is an option $[3,7,12,26]$. When doing sequestrectomy quite often soft tissue debridement is unavoidable for infection eradication then eventually there may be uncovered bone and the wound will heal by secondary intention thanks to serial wound care using different chemicals and technique varying from settings, the intention is to eradicate the infection and the wound heal by secondary intention. Once infection cleared and skin or soft tissue defect have remained a concern, then skin graft or different flaps may be done [31].

\section{Chronic Suppressive Antibiotherapy (CSAT)}

Postoperative antibiotherapy is an adjunct to thorough sequestrectomy or administered alone in case operation is not possible or available. Ideally the antibiotics should target a specifically identified and sensitive organism. Postoperatively parenteral antibiotics are administered for 6 weeks; 2 to 3 months of oral antibiotics are recommended, but the time cut off maybe directed by inflammatory markers such as ESR and CRP $[3,28]$.

\section{Discussion}

Chronic osteomyelitis remains a very difficult orthopaedic challenge in SSA as well as other LICs, contrary to the western world where earlier diagnosis at an acute or subacute stage coupled with meticulous surgical technique minimize COM rate. The difference in incidence from Lowand High-Income countries does not only lie on surgical yield but also on difference in patient's characteristics and associated co-morbidities. In SSA there is a dearth of epidemiological studies reporting the incidence of COM; the only report noted 43-200/100000 in LIC and it focused on the paediatric population [11]. The tibia at 30 to $35 \%$ of all cases of COM is the most frequently affected bone. The tibia is subcutaneous anteriorly and not fully covered by muscles and thus more prone to open fractures. COM in SSA is highly prevalent in the paediatric population with a peak incidence in second decade of life [5,13-16].

Sickle cell disease, peripheral vascular disease and diabetes mellitus are potential risk factors of COM in SSA and are known to be a global factor and often resulting into poly-microbial infections. Immunosuppression resulting from malnutrition is a documented potential risk factor of COM in SSA, with relatively higher level of evidence [25-27]. Buruli ulcers are as well a distinct risk factor related to SSA COM. Staphylococcus Aureus at 60 to $80 \%$ (with higher level of evidence) is responsible for COM, as it is known to be a normal skin flora $[12,14,29]$. Moreover, septicaemia was highlighted to be a risk factor for hematogenous osteomyelitis.

Chronic osteomyelitis may present as hematogenous or exogenous, the later can be post-traumatic or post-operative. Thus, its clinical 
presentation may vary depending on patient characteristics, location of the disease, and chronicity of symptoms. The main clinical presentations are sinus drainage at $50-93 \%$, bone pain, limb deformity, pathological fractures, bone overgrowth and joint stiffness. The holistic way of systemic and exhaustive clinical examination is still applicable and highly recommended when COM is suspected. Convention radiography and CT imaging are gold standard adjuncts to the clinical examination; the role of ultrasound is limited to soft tissue extension of the disease and sub-periosteal abscess. The role of MRI in COM for diagnosis is still controversial and infrequently utilized in SSA for lack of availability expense reasons. MRI can be of use in the planning of therapeutic procedures.

COM in SSA continues to be prevalent. some Effective and available therapeutic measures do exist; there is a need for harmonizing evidencebased practices via systems of care and 'best practice' protocols tailored to one's locale and environment. Treatment can have a curative or suppressive/palliative intent. The curative treatment consists of combined operative and medical management. The four components for effective treatment of COM include: surgical infection control, dead space management, bone and soft tissue reconstruction in case of tissue loss and chronic adjunctive antibiotics [3,30]. Appropriate diagnosis, triage of the patient, and timing and preparation for a technically sound operation are the keys in management of patients with COM. One goal is to avoid unnecessary reconstructive procedures projecting a poor outcome.

Chronic osteomyelitis continues to be prevalent in SSA, more recently, a shift from hematogenous to exogenous osteomyelitis has been observed, likely secondary to the prevalence of vehicular trauma and fracture treatment utilizing open reduction and internal fixation utilizing osteostynthetic material use. The present study highlights common risk factors, incidences, and management options in SSA. Very limited data of high quality on COM in SSA currently exist, reporting its epidemiology and socio-economic burden it poses. Further research, trauma registries to assess the epidemiology, outcomes, economic burden, and diagnostic and management protocols for this common condition in Sub-Saharan Africa are needed.

\section{Acknowledgement}

The authors acknowledge the editorial input and assistant from MJ Tarpley and JL Tarpley plus the contributions of Dr. Alan Bennie.

\section{Disclosure statement}

We declare no conflict of interest regarding this systematic review.

\section{References}

1. Jorge LS, Chueire AG, Rossit AR (2010) Osteomyelitis: a current challenge. Braz J Infect Dis 14: 310-315. [Crossref]

2. Walter G, Kemmerer M, Kappler C, Hoffmann R (2012) Treatment Algorithms for Chronic Osteomyelitis. Dtsch Arztebl Int 109: 257-64. [Crossref]

3. Ikpeme IA, Ngim NE, Ikpeme AA (2010) Diagnosis and treatment of pyogenic bone infections. Afr Health Sci 10: 82-88. [Crossref]

4. Jorge LS, Chueire AG, Fucuta PS, Machado MN, Oliveira MGL, et al. (2017) Predisposing factors for recurrence of chronic posttraumatic osteomyelitis: a retrospective observational cohort study from a tertiary referral center in Brazil. Patient Saf Surg 11: 17. [Crossref]

5. Agaja SB, Ayorinde RO (2008) Chronic osteomyelitis in Ilorin, Nigeria. S Afr J Surg 46: 116-118. [Crossref]

6. Li Q, Cui H, Dong J, He Y, Zhou D, et al. (2015) Squamous cell carcinoma resulting from chronic osteomyelitis: a retrospective study of 8 cases. Int J Clin Exp Pathol 8: 10178-10184. [Crossref]
7. Biruk WL, Wubshet K (2007) Chronic Osteomyelitis at Tikur Anbessa Hospital, Addis Ababa University, Ethiopia. East Cent Afr J Surg 12.

8. Kremers HM, Nwojo ME, Ransom JE, Wood-Wentz CM, Melton LJ 3rd, et al. (2015) Trends in the epidemiology of osteomyelitis: a population-based study, 1969 to 2009. J Bone Joint Surg Am 97: 837-845. [Crossref]

9. Onche II, Obiano SK (2004) Chronic osteomyelitis of long bones: reasons for delay in presentation. Niger J Med 13: 355-358. [Crossref]

10. Bratzler DW, Dellinger EP, Olsen KM, Perl TM, Auwaerter PG, et al. (2013) Clinical practice guidelines for antimicrobial prophylaxis in surgery. Am J Health-Syst Pharm 70: 195-283. [Crossref]

11. Dartnell J, Ramachandran M, Katchburian M (2012) Haematogenous acute and subacute paediatric osteomyelitis A systematic review of the literature. J Bone Jt Surg $\operatorname{Br}$ 94: 584-595. [Crossref]

12. Beckles VL, Jones HW, Harrison WJ (2010) Chronic haematogenous osteomyelitis in children: a retrospective review of 167 patients in Malawi. J Bone Joint Surg Br 92: 1138-1143. [Crossref]

13. Stanley CM, Rutherford GW, Morshed S, Coughlin RR, Beyeza T (2010) Estimating the healthcare burden of osteomyelitis in Uganda. Trans R Soc Trop Med Hyg 104: 139-142. [Crossref]

14. Ouedraogo S, Zida M, Walla A, Tall M (2017) Epidemiological, bacteriological and therapeutic aspects of chronic osteomyelitis in a sub-Saharan environment. Med Sante Trop 27: 292-295. [Crossref]

15. Ibingira CB (2003) Chronic osteomyelitis in a Ugandan rural setting. East Afr Med J 80: 242-246. [Crossref]

16. Ali AM, Maya E, Lakhoo K (2014) Challenges in managing paediatric osteomyelitis in the developing world: Analysis of cases presenting to a tertiary referral centre in Tanzania. Afr J Paediatr Surg 11:308-11. [Crossref]

17. Bickler SW, Rode H (2002) Surgical services for children in developing countries. Bull World Health Organ 80: 829-835. [Crossref]

18. Bahebeck J, Atangana R, Techa A, Monny-Lobe M, Sosso M, et al. (2004) Relative rates and features of musculoskeletal complications in adult sicklers. Acta Orthop Belg 70: 107-111. [Crossref]

19. Panteli M, Giannoudis PV (2017) Chronic osteomyelitis: what the surgeon needs to know. EFORT Open Rev 1: 128-135. [Crossref]

20. Hatzenbuehler J, Pulling TJ (2011) Diagnosis and management of osteomyelitis. Am Fam Physician 84: 1027-33. [Crossref]

21. Pommelet V, Vincent QB, Ardant MF, Adeye A, Tanase A, et al. (2014) Findings in patients from Benin with osteomyelitis and polymerase chain reaction- confirmed mycobacterium ulcerans infection. Clin Infect Dis 59: 1256-1264. [Crossref]

22. Nwadiaro HC, Ugwu BT, Legbo JN (2000) Chronic osteomyelitis in patients with sickle cell disease. East Afr Med J 77: 23-26. [Crossref]

23. Thanni LO (2006) Bacterial osteomyelitis in major sickling haemoglobinopathies: geographic difference in pathogen prevalence. Afr Health Sci 6: 236-239. [Crossref]

24. Mthethwa PG, Marais LC (2017) The microbiology of chronic osteomyelitis in a developing world setting. SA Orthop J 16: 39-45.

25. Beckles VL, Jones HW, Harrison WJ (2011) Chronic haematogenous osteomyelitis in children: A retrospective review of 167 patients in Malawi. J Bone Jt Surg Br 92: 113843. [Crossref]

26. Nwagbara IC, Opara KO (2017) Chronic Osteomyelitis of the Long Bones. Orient J Med 29: 3-4.

27. Gashegu J, Byamungu T, Ngarambe C, Bayisenga J, Kiswezi A (2018) Treatment of chronic osteomyelitis with locally made calcium sulfate bone cement pellets impregnated with antibiotics at University Teaching Hospital of Butare (CHUB), Rwanda. East Cent Afr J Surg 23: 1.

28. Marais LC, Ferreira N, Aldous C, Le Roux TL (2016) The outcome of treatment of chronic osteomyelitis according to an integrated approach. Strategies Trauma Limb Reconstr 11: 135-142. [Crossref]

29. Orimolade EA, Salawu L, Oginni LM (2007) Clinical and laboratory features of Nigerian patients with osteomyelitis. Singap Med J 48:917-921. [Crossref] 
30. Marais LC, Ferreira N, Le Roux TL (2014) The management of chronic osteomyelitis: Part I - Diagnostic work-up and surgical principles. SA Orthop J 13: 2.

31. Baldan M, Gosselin RA, Osman Z, Barrand KG (2014) Chronic osteomyelitis management in austere environments: the International Committee of the Red Cross experience. Trop Med Int Health 19: 832-837. [Crossref]
32. Museru LM, Mcharo CN (2001) Chronic osteomyelitis: a continuing orthopaedic challenge in developing countries. Int Orthop 25: 127-131. [Crossref]

33. Alonge TO, Ogunlade SO, Omololu AB, Fashina AN, Oluwatosin A (2002) Management of chronic osteomyelitis in a developing country using ceftriaxonePMMA beads: an initial study. Int J Clin Pract 56: 181-183. [Crossref]

\section{Appendix}

Level I: Large well conducted Randomized Controlled Clinical trials, Systematic review and Meta-analyses

Level II: Small well conducted Randomized Controlled Clinical trials

Level III: Well conducted cohort study

Level IV: Well conducted Case Control studies

Level V: Uncontrolled studies that were not well conducted.

Level VI: Conflicting evidence that tends favor the recommendations

Level VII: Expert opinion or data extrapolated from evidence for general principles and other procedures

Copyright: (2019 Olivier K. This is an open-access article distributed under the terms of the Creative Commons Attribution License, which permits unrestricted use, distribution, and reproduction in any medium, provided the original author and source are credited. 